\title{
NOUVELle
}

\section{Les usines virales rabiques se forment dans la cellule par séparation de phase liquide}

Jovan Nikolic, Cécile Lagaudrière-Gesbert, Nathalie Scrima, Danielle Blondel, Yves Gaudin
Institut de biologie intégrative de la cellule (I2BC), CEA, CNRS, université Paris-Sud, université Paris-Saclay,

91198, Gif-sur-Yvette Cedex, France.

yves.gaudin@i2bc.paris-saclay.fr
> La réplication et l'assemblage de nombreux virus se déroulent dans des compartiments intra-

cellulaires spécialisés nommés usines virales [1] $(\rightarrow)$. Ces organites for- $(\rightarrow)$ Voir la Nouvelle de J. Nikolic et D. Blondel, $\mathrm{m} / \mathrm{s}$ $n^{\circ} 11$, novembre 2017, page 921 més durant l'infection concentrent les protéines et acides nucléiques viraux mais également des facteurs cellulaires nécessaires à la réplication virale. Ces usines semblent aussi limiter la reconnaissance des acides nucléiques viraux par les protéines senseurs de l'immunité innée, et restreindre l'accès des protéines cellulaires antivirales à la machinerie de réplication [2].

\section{Diversité des usines virales}

La localisation et la nature des usines virales sont très diverses. Elles dépendent du génome viral (ADN ou ARN) et de la stratégie de réplication du virus. Les premières usines caractérisées ont été celles des gros virus à ADN comme les poxviridae (virus de la variole et de la vaccine). Ces usines, dépourvues de membrane, sont localisées au voisinage du centre organisateur des microtubules, elles sont entourées d'une cage de vimentine et recrutent les mitochondries; elles contiennent également de nombreuses protéines chaperons et des protéines de choc thermique [3]. Dans le cas des virus dont le génome est un ARN de polarité positive (c'est-à-dire de même polarité que les ARN messagers), les usines virales sont associées à des réarrangements de diverses membranes cellulaires (réticulum endoplasmique, mitochondrie, etc.) ; l'intérieur de ces usines est en connexion avec le cytoplasme par des canaux qui permettent l'entrée de ribonucléotides et l'export des ARN synthétisés [4].

Les virus à $A R N$ négatif simple brin (ordre des Mononegavirales) induisent aussi la formation d'inclusions cytoplasmiques qui sont également des usines virales dans lesquelles sont synthétisés les ARN viraux [5-7]. Le cas le plus emblématique est celui du virus de la rage dont les inclusions, identifiées pour la première fois par Aldrich Negri en 1903 [8] et baptisées corps de Negri, peuvent atteindre plusieurs microns de diamètre.

\section{Le cycle du virus de la rage}

Le virus de la rage est un virus neurotrope qui cause une encéphalite fatale chez les mammifères et qui tue encore plus de 50000 personnes chaque année [9] $(\rightarrow)$.

$(\rightarrow)$ Voir la Nouvelle de F. RibadeauDumas et al., $\mathrm{m} / \mathrm{s}$ $n^{\circ} 1$, janvier 2013 , page 47

Son génome de polarité négative code cinq protéines. La nucléoprotéine (N) s'assemble tel un manchon autour de I'ARN génomique pour former la nucléocapside qui est associée à l'ARN-polymérase $A R N$-dépendante $(L)$ et à son cofacteur, la phosphoprotéine $(P)$. L'ensemble constitue la ribonucléoprotéine (RNP) qui est enveloppée d'une membrane provenant de la cellule hôte. Dans cette membrane, on trouve une unique glycoprotéine ( $G$ ) qui va permettre les étapes d'entrée du virus dans la cellule hôte. Enfin, sur la face interne de la membrane, se trouve la protéine de matrice (M) qui forme un pont entre la RNP condensée sous forme hélicoïdale et la membrane virale. Le virion pénètre dans la cellule par une étape d'endocytose qui est suivie de la fusion de sa membrane avec celle de l'endosome pour libérer la RNP dans le cytoplasme. La RNP va servir de matrice pour la transcription des ARN messagers viraux par la polymérase L. Ces ARN viraux sont traduits en protéines par les ribosomes cellulaires puis, lorsque suffisamment de nouvelles protéines virales ont été synthétisées, la polymérase virale bascule dans la phase de réplication. Elle synthétise alors des antigénomes (de polarité positive), copie complémentaire du génome entrant. Ces antigénomes sont encapsidés par la protéine $\mathrm{N}$ au fur et à mesure de leur synthèse. Ils servent ensuite de matrice pour la synthèse de génomes nouveaux. Ces génomes peuvent euxmême servir de matrice dans une nouvelle étape de transcription ou bien être compactés et aller bourgeonner au niveau d'une membrane cellulaire pour former de nouveaux virions.

Les usines virales formées par le virus de la rage sont des organites liquides Nous avions montré que toutes les étapes de synthèse d'ARN (qu'ils soient messagers, antigénomiques ou génomiques) se déroulent au sein des corps de Negri qui concentrent aussi les protéines de la machinerie de réplication (L, N et P) [6]. Toutefois, la nature physico-chimique et les principes 
A
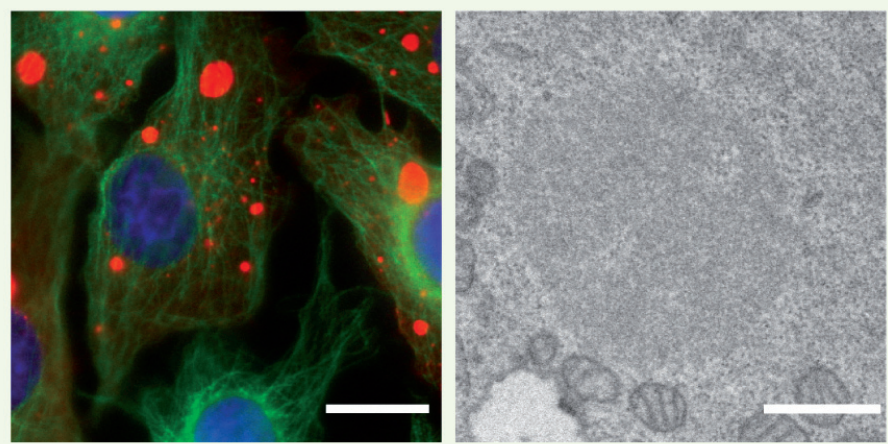

B

Figure 1. Détection du virus de la rage par immunodétection et microscopie électronique. A. Cellules en culture infectées par le virus de la rage et observées en microscopie optique à fluorescence. Les noyaux des cellules sont en bleu. Les usines virales sont constituées par les moyennes et grosses inclusions circulaires en rouge. Le réseau de microtubules est en vert. C'est le long de ce réseau que sont transportées les nucléocapsides virales (petits points rouges) lorsqu'elles quittent l'usine virale. Barre d'échelle : $15 \mu \mathrm{m}$. B. Corps de Negri observé en microscopie électronique. II présente une structure granulaire dense aux électrons et est entouré de mitochondries. Barre d'échelle : $1 \mu \mathrm{m}$.

permettant la morphogenèse de ces usines virales dépourvues de membrane étaient inconnus. La sphéricité de ces structures suggérait néanmoins qu'elle pouvait appartenir à une catégorie d'organites récemment découverte : les organites liquides qui se forment dans le cytosol par séparation de phase.

Pour examiner la nature liquide des corps de Negri, nous avons infecté des cellules par un virus recombinant exprimant la protéine $P$ fusionnée à une protéine fluorescente [10]. Nous avons ainsi pu suivre, par vidéomicroscopie, la morphogenèse et la dynamique des corps de Negri. Nous avons montré que, dans la cellule infectée, ils sont capables de fusionner entre eux et de se déformer de façon réversible lorsqu'ils rencontrent une barrière physique. Des expériences de recouvrement de fluorescence après photoblanchiment ont définitivement permis de démontrer leur nature liquide, mais indiquent également que la phosphoprotéine $P$ est capable de faire la navette entre le cytosol et l'intérieur des usines virales. Enfin, nous avons observé que les corps de Negri sont sensibles à leur environnement physico-chimique: un choc hypoto- nique conduit à leur rapide et complète disparition de la cellule infectée suivie de leur réapparition après une dizaine de minutes. Les films obtenus par vidéo-microscopie révèlent également que des génomes compactés sont expulsés des usines virales et qu'ils sont ensuite transportés le long du réseau des microtubules pour former éventuellement de nouvelles usines [10].

II a été possible d'établir un système minimal reproduisant toutes les caractéristiques des corps de Negri [10]. Ce système s'appuie sur l'expression transitoire des seules protéines virales $\mathrm{N}$ et $P$ dans le cytoplasme de la cellule en dehors du contexte de l'infection. Une série de mutations nous a permis de caractériser les régions de la protéine $P$ qui sont nécessaires pour la formation de ces organites liquides. Le domaine d'interaction de $P$ avec la protéine $N$ doit ainsi être présent, c'est-à-dire que l'interaction directe entre les deux protéines est nécessaire pour que la séparation de phase se produise. Par ailleurs, un domaine intrinsèquement désordonné de $\mathrm{P}$ s'est lui aussi révélé indispensable. Il est très probable que, comme cela a été proposé pour d'autres organelles liquides, les acides aminés de ces domaines très flexibles permettent la formation d'interactions faibles entre protéines virales, interactions faibles qui sont à l'origine de la séparation de phase liquide.

\section{Conclusions}

La formation d'usine ayant des propriétés d'organites liquides par séparation de phase permet au virus de conserver son identité au sein de la cellule infectée et de distinguer ce qui le constitue (l'usine virale) de ce qui lui est extérieur (le reste du cytoplasme). Le caractère liquide des corps de Negri semble d'ailleurs pouvoir être étendu aux usines virales de nombreux autres Mononegavirales tels que les Filovirus (comme le virus Ebola) ou les Paramyxovirus (comme le virus de la rougeole).

La question se pose alors de savoir si l'immunité innée de la cellule est capable de détecter ces compartiments pour combattre l'agresseur ou si, au contraire, ceux-ci permettent au virus de passer inaperçu. En particulier, les étapes de transcription du génome viral conduisent à la formation d'ARN double brin possédant parfois des extrémités 5' triphosphate. Ce type de motifs moléculaires (ou PAMP pour pathogen-associated molecular pattern), que l'on devrait donc retrouver essentiellement au sein de l'usine virale, est la signature d'une infection par un virus à ARN. Certaines protéines cellulaires sont d'ailleurs susceptibles de les détecter pour ensuite aller activer l'immunité innée. De façon remarquable, l'infection rabique induit la formation de granules de stress qui sont eux aussi des organites liquides très dynamiques et enrichis en protéines capables de détecter les PAMP [1]. Néanmoins, ils forment une phase liquide dont nous avons montré qu'elle n'est pas miscible avec celle des corps de Negri [10]. Le problème de la détection des PAMP par les senseurs de l'immunité innée reste donc entier. 


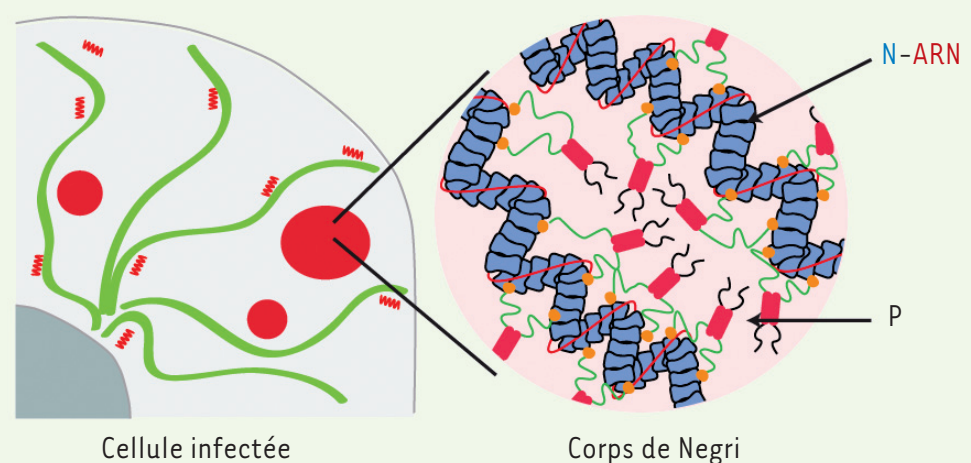

Cellule infectée

Corps de Negri

Figure 2. Schématisation d'un corps de Negri. Dans la cellule infectée, cohabitent quelques gros corps de Negri en rouge (un ou deux par cellule qui se sont formés autour des génomes des virus entrants), des corps de Negri de taille moyenne (formés autour des génomes synthétisés dans la cellule infectée) et des nucléocapsides isolées. Les corps de Negri contiennent essentiellement des nucléocapsides ( $\mathrm{N}-\mathrm{ARN}$ ) et de la phosphoprotéine P. La cohésion de la phase liquide est probablement assurée par des interactions faibles entre les domaines intrinsèquement désordonnés de $P$.

Enfin, la découverte du caractère liquide des usines virales ouvre de nouvelles pistes thérapeutiques. Des drogues capables de disperser la phase liquide les constituant devraient être efficaces contre l'infection. $\diamond$

Rabies virus factories are formed by liquid-liquid phase separation

\section{LIENS D'INTÉRÊT}

Les auteurs déclarent n'avoir aucun lien d'intérêt concernant les données publiées dans cet article.

NOUVELLE

\section{Un point de contrôle développemental synchronise la morphogenèse et la différenciation cellulaire dans l'embryon de mammifère}

Julien G. Dumortier, Jean-Léon Maître

> Pendant le développement embryonnaire, la destinée des cellules est déterminée par les informations sur leurs positions dont elles disposent. La différenciation cellulaire déclenche souvent des modifications dans l'organisation de l'embryon qui, en retour, créent de nouvelles informations de positionnement. Ces cycles de morphogénèse et de différenciation sont des processus indis-

\section{RÉFÉRENCES}

1. Nikolic J, Blondel D. Le virus de la rage induit la formation de granules de stress en contact étroit avec les usines virales. Med Sci (Paris) 2017 ; 33 : 921-3.

2. Netherton $\mathrm{CL}$, Wileman T. Virus factories, double membrane vesicles and viroplasm generated in animal cells. Curr Opin Virol $2011 ; 1$ : 381-7.

3. Schramm B, Locker JK. Cytoplasmic organization of POXvirus DNA replication. Traffic $2005 ; 6$ : 839-46.

4. Harak C, Lohmann V. Ultrastructure of the replication sites of positive-strand RNA viruses. Virology 2015 ; 479-480: 418-33.

5. Hoenen T, Shabman RS, Groseth A, et al. Inclusion bodies are a site of ebolavirus replication. J Virol $2012 ; 86$ : 11779-88.

6. Lahaye X, Vidy A, Pomier C, et al. Functional characterization of Negri bodies (NBs) in rabies virusinfected cells: evidence that NBs are sites of viral transcription and replication. J Virol 2009 ; $83: 7948-58$

7. Rincheval V, Lelek M, Gault $\varepsilon$, et al. Functional organization of cytoplasmic inclusion bodies in cells infected by respiratory syncytial virus. Nat Commun $2017 ; 8: 563$

8. Negri A. Contributo allo studio dell' eziologia della rabia. Bol Soc Med Chir Pavia 1903 ; 2 : 88-114.

9. Ribadeau-Dumas F, Dacheux L, Bourhy H. La rage. Med Sci (Paris) $2013 ; 29: 47-55$.

10. Nikolic J, Le Bars R, Lama Z, et al. Negri bodies are viral factories with properties of liquid organelles. Nat Commun $2017 ; 8: 58$.

Unité génétique et biologie du développement, Institut Curie, PSL Research University, CNRS UMR3215, Inserm U934, 75248 Paris, France. jean-leon.maitre@curie.fr

pas atteints. Une étude de Shahbazi et ses collègues, parue récemment dans Nature [1], révèle les mécanismes d'un point de contrôle développemental qui agit durant les tout premiers jours de l'embryon du mammifère.

Chez de nombreux mammifères, aux stades initiaux du développement, l'embryon forme une structure, appelée blastocyste, qui s'implante dans l'utérus 